\title{
MODELLING OF MAGNETIC HYPERFINE FIELD IN NANOPARTICLES
}

\author{
K. Mažeika, A. Amulevičius, and D. Baltrūnas \\ Institute of Physics, Savanoriu 231, LT-02300 Vilnius, Lithuania \\ E-mail: kestas@ar.fi.lt
}

Received 15 March 2006

\begin{abstract}
In order to obtain the information on the dynamics of magnetic moments from Mössbauer spectra of nanostructured magnetic materials one should perform a complicated description of the spectra. The possibilities of a many-state relaxation model for the description of Mössbauer spectra are studied. The many-state relaxation model is applied to explain the dependence of spectra of nanoparticles in ferrofluids on temperature. For the complicated Mössbauer spectra which can have many static subspectra, the simulation of the average hyperfine field is used. For the description of dependence of the hyperfine field on temperature the many-state model can be used to obtain the temperature dependence of magnetic anisotropy or other parameters that affect the rate of relaxation.
\end{abstract}

Keywords: Mössbauer spectroscopy, nanoparticles, magnetic hyperfine field, relaxation, modelling

PACS: $76.80 .+\mathrm{y}, 75.75 .+\mathrm{a}, 76.60 . \mathrm{Es}$

\section{Introduction}

Magnetic nanostructures and nanoparticles have recently been intensively studied $[1,2]$. The nanosized materials have different properties compared with the bulk materials. The properties differ because of the small sizes of structures and, as a consequence, a large relative amount of surface or interface atoms. One class of magnetic nanocrystalline materials is magnetic fluids or ferrofluids, where ferromagnetic or ferrimagnetic nanoparticles are dispersed in the carrier fluid. Magnetic fluids (as well as solid nanocrystalline systems) have different concentration of the nanostructured component - nanoparticles. Depending on the concentrations and how the particles are ordered, the interactions between the particles differ. The magnetic dipole, exchange, as well as non-magnetic Van der Waals, etc. interactions may be important. However, their influence on differently diluted nanocrystalline systems is still poorly understood.

One of the methods frequently used for the studies of such magnetic materials is Mössbauer spectroscopy. However, for the systems which consist of small particles the influence of relaxation of the magnetic moment is important. In this case the description of Mössbauer spectra by the relaxation models is required. Here the many-state relaxation model is applied to the description of dependence of the average hyperfine field of complicated Mössbauer spectra. Another case which is considered in this study is the investigation of possibility of using the model for the relaxed Mössbauer spectrum when the external magnetic field is applied.

\section{The relaxation problem in the systems of nanoparticles}

Mössbauer spectroscopy of ${ }^{57} \mathrm{Fe}$ provides information on the atomic state of material. Such possibility is based on the sensitivity of Mössbauer spectra to the hyperfine interactions between the atom, its surroundings, and the nucleus. Hyperfine magnetic interactions cause magnetic splitting of spectra (sextets) in the materials where ferro-, ferri-, and antiferromagnetic order exists. The splitting of nuclear levels and magnetic structure of Mössbauer spectra is determined by the interactions between a nucleus and an atom as well as a nucleus and the external magnetic field. Therefore, the magnetic interaction Hamiltonian may be given by [3]

$$
\mathcal{H}=\mathbf{I} \mathbf{A} \mathbf{S}+g \beta \mathbf{I} \mathbf{H}_{\mathrm{e}},
$$

where $\mathbf{I}$ is the nuclear spin, $\mathbf{S}$ is the spin of an atom, $\beta$ is the nuclear magneton, $g$ is the nuclear $g$-factor, and $\mathbf{H}_{\mathrm{e}}$ is the external magnetic field. The hyperfine interaction term is expressed by the hyperfine interaction tensor A. However, instead of it the magnetic hyperfine 
field $\mathbf{H}_{\mathrm{i}}=\mathbf{A}\langle\mathbf{S}\rangle /(g \beta)$ and the expression of Hamiltonian

$$
\mathcal{H}=g \beta \mathbf{I}\left(\mathbf{H}_{\mathrm{i}}+\mathbf{H}_{\mathrm{e}}\right)
$$

are most frequently used. The time dependence of the hyperfine magnetic interaction is taken into account. $\langle\mathbf{S}\rangle$ is the average of spin $\mathbf{S}$ of an atom over the nuclear sensing time $\tau_{\mathrm{s}}$. In this way the dynamic changes in magnetic hyperfine fields, which affect Mössbauer spectra, occur. Therefore, Mössbauer spectroscopy allows studying the time-dependent phenomena on an atomic scale, such as fluctuations of the magnetic moment of nanoparticles. For the most commonly used ${ }^{57} \mathrm{Fe}$ isotope, the frequency of fluctuations of $\langle\mathbf{S}\rangle$, where considerable changes in the hyperfine field $\mathbf{H}_{\mathrm{i}}$ take place, is approximately within $10^{7}-10^{9} \mathrm{~s}^{-1}$. The upper limit is determined by the Mössbauer nuclear lifetime $\tau_{\mathrm{N}}$. The fluctuations of the magnetic moment cause a considerable broadening of the lines of spectra when their frequency is above $1 / \tau_{\mathrm{N}}$. When the frequency is approaching $1 / \tau_{\mathrm{s}}$, the magnetic structure of a sextet disappears - it collapses to a singlet. For the description of collapsed Mössbauer spectra, when fluctuations of the magnetic moment of single-domain nanoparticles are fast, the model of two states is most frequently used. It takes into account only two states of the minimal energy [4]. These states are separated by the barrier of the height $\Delta E$. If $\Delta E$ is due to magnetic anisotropy, the time of jumps between these states can be evaluated according to the Brown formula $[5,6]$

$$
\tau=\tau_{0} \exp \left(\frac{K V}{k_{\mathrm{B}} T}\right) .
$$

$\tau_{0}$ is the exponential prefactor,

$$
\tau_{0}=\frac{M_{s} \sqrt{\pi}}{K \gamma}\left(\frac{K V}{k_{\mathrm{B}} T}\right)^{-1 / 2},
$$

where $k_{\mathrm{B}}$ is the Boltzmann constant. Time between the jumps $\tau$ depends on the energy barrier height $\Delta E=$ $K V$ and temperature $T . M_{s}$ is magnetization, $K$ is the anisotropy constant, $V$ is the volume of nanoparticles, and $\gamma$ is the gyromagnetic ratio. $\tau_{0}$ depends on temperature very little compared with the exponential and is usually within $10^{-9}-10^{-11} \mathrm{~s}$.

The relaxational model of two states is used when the collapsed Mössbauer spectrum is described by a singlet. The model of two states takes into account only two opposite orientations of the magnetic moment of the particle which are collinear with the easy magnetization axis. The model cannot describe the influence of deviations of direction of the magnetic moment from the easy magnetization axis to Mössbauer spectra. When the small angle deviations from easy magnetization directions are sufficiently important, the changes in spectra can be described using the linear decrease in the hyperfine field with temperature [3]

$$
H_{\mathrm{i}}=H_{0}\left(1-\frac{k_{\mathrm{B}} T}{2 K V}\right)
$$

where $H_{0}$ is the hyperfine field for the bulk material.

Because of the volume distribution of particles, Mössbauer spectra are sums of collapsed (paramagnetic) and noncollapsed (magnetic) parts. If nanoparticles undergo rapid magnetic transition to the superparamagnetic state, Eq. (5) and the broadened Lorentzian singlet give good description of Mössbauer spectra. Otherwise, if the transitional spectra are important, the description of spectra may be insufficiently correct. In such cases, it is better to use a many-state model [7]. All kinds of fluctuations of the magnetic moment around the easy magnetization axis are taken into account here. The many-state model can be used for the description of Mössbauer spectra within the studied temperature range. This model is good even if the transition of magnetic material to the superparamagnetic state is slow and the transitional spectra (non-Lorentzian shape spectra) are important in a wide temperature region. The relaxation in the many-state model is defined by the dependence of energy on the direction of the magnetic moment and the relaxation rate between the states. Therefore, different magnetic energy dependences on the angle are possible, among them those obtained for the particles which interact with other particles or the external magnetic field.

The use of the many-state model requires large matrix inversion. Despite that, the experimental Mössbauer spectra are recently successfully described by the many-state model using the least-squares procedure $[8,9]$. On the other hand, for the Mössbauer spectra which have many static subspectra, such description is still complicated. The application of the many-state model may be much more complicated when other hyperfine interactions and the interaction between particles are important and because of that the Liouville operators are needed. 


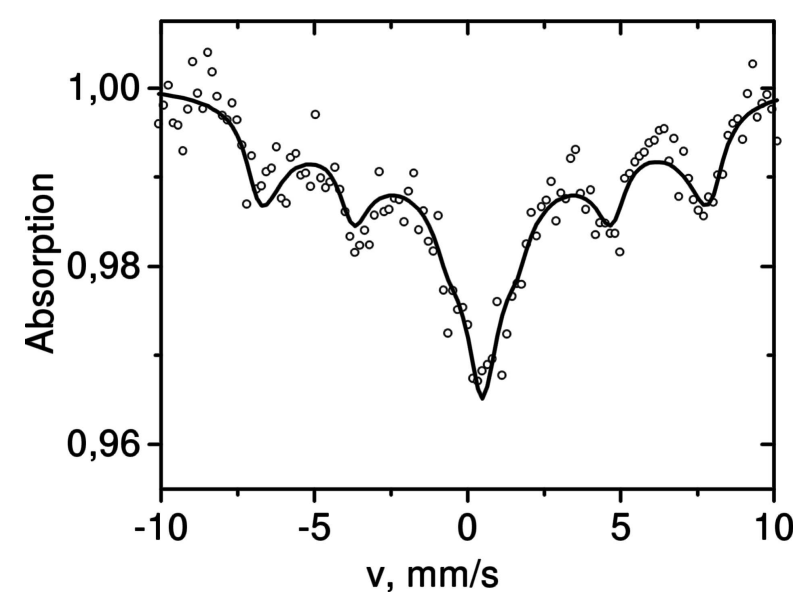

Fig. 1. Mössbauer spectra of $\mathrm{Zn}_{0.7} \mathrm{Co}_{0.3} \mathrm{Fe}_{2} \mathrm{O}_{4}$ particles with the mean diameter of $5.2 \mathrm{~nm}$ described by the many-state model at $T=83 \mathrm{~K}$.

\section{Application of the many-state relaxation model}

\subsection{Many-state model}

The many-state relaxation model is generalization of a stochastic model given by the Hamiltonian [10]

$$
\mathcal{H}(t)=g \beta h I_{z} f(t),
$$

where the magnetic field randomly varies between $\pm h$ as described by the random function $f(t)= \pm 1$ (the two-state model). $I_{z}$ is the component of the nuclear spin along the $z$ axis. The many-state model is described similarly, but here the magnetic field has many intermediate values.

The Hamiltonian of Eq. (6) describes the influence of the random magnetic hyperfine field on the energy of the states of a nucleus. The contributions of other hyperfine interactions (quadrupole) or the interaction with the external magnetic field may also be important. These contributions can change time properties of the Hamiltonian. If the Hamiltonian commutes with itself at different times, as given by Eq. (6), its time variation will not cause the transitions between the nuclear states. Then the description of influence of the magnetic hyperfine field variation can be separated for each line of Mössbauer spectra and Liouville operators are not needed. Though the many-state relaxation model has recently become sufficiently practical using personal computers for the description of Mössbauer spectra using the least-squares procedure, the application of Liouville operators makes the use of the many-state model much more complicated and it has not been implemented yet [9].

When the interaction of a nucleus with the hyperfine field $H_{\mathrm{i}}$ is stronger than the quadrupole interaction and there are no other interactions, the Hamiltonian can be approximated in the form of Eq. (6). In such a case the shape of each line of the Mössbauer spectra may be given by [7]

$$
I(\omega)=2 \operatorname{Re}\left(\mathbf{W M}^{-1} \mathbf{1}\right),
$$

where elements of $\mathbf{W}$ matrix are probabilities of population of states. Matrix

$$
\mathbf{M}=(\mathrm{i} \omega+\Gamma) \underline{\mathbf{I}}-\mathrm{i} \boldsymbol{\Omega}-\mathbf{\Pi}
$$

describes the system using the position of lines of Mössbauer spectra (elements of matrix $\Omega$ ) and the natural width of lines $\Gamma$. The matrix $\Pi$ is expressed by the probabilities of jumps between the states of the electronic system. These states correspond to different values of the hyperfine field that is directed along the easy magnetization axis.

Thus, applying two parameters, the relaxation rate $R$ and the energy barrier height $\Delta E$, the Mössbauer spectra can be simulated (Fig. 1). There is a relation between the relaxation rate $R$ and $\tau_{0}$ in Eq. (3) [7]:

$$
R=\frac{\sqrt{2 \pi}}{\tau_{0}}\left(\frac{2 K V}{k_{\mathrm{B}} T}\right)^{-3 / 2} .
$$

The real assembly of nanoparticles has particles of different sizes. Due to the size distribution the energy barrier height is also distributed. The size distribution of nanoparticles is usually described by the lognormal law

$$
f(D)=\frac{1}{D} \exp \left(-\frac{\ln ^{2} \frac{D}{D_{0}}}{2 \sigma^{2}}\right),
$$

where $D_{0}$ is the average size of particles and $\sigma$ is its standard deviation. For the description of experimental Mössbauer spectra, the parameters of size distribution and the static parameters (isomer shift, line width, magnetic and quadruapole splittings) of Mössbauer spectra should be included. The example of such description for $\mathrm{Zn}_{0.7} \mathrm{Co}_{0.3} \mathrm{Fe}_{2} \mathrm{O}_{4}$ nanoparticles is shown in Fig. 1 . For the spectrum the relaxation time $\tau=6 \cdot 10^{-9} \mathrm{~s}$ is determined on the basis of Eqs. (3), (4), and (9).

For the simulation of artificial Mössbauer spectra, a very large number of states (large matrixes in Eq. (8)) is not needed. The total number of states in the model is determined by the spin $S$, which has meaning of the total spin of the particle, but here it is the parameter of simulation. The hyperfine field jumps between the values which are proportional to $S_{z}$, the projection of $\mathbf{S}$, and the total number $n=2 S+1$ of states is obtained. When the states are energetically close, the 


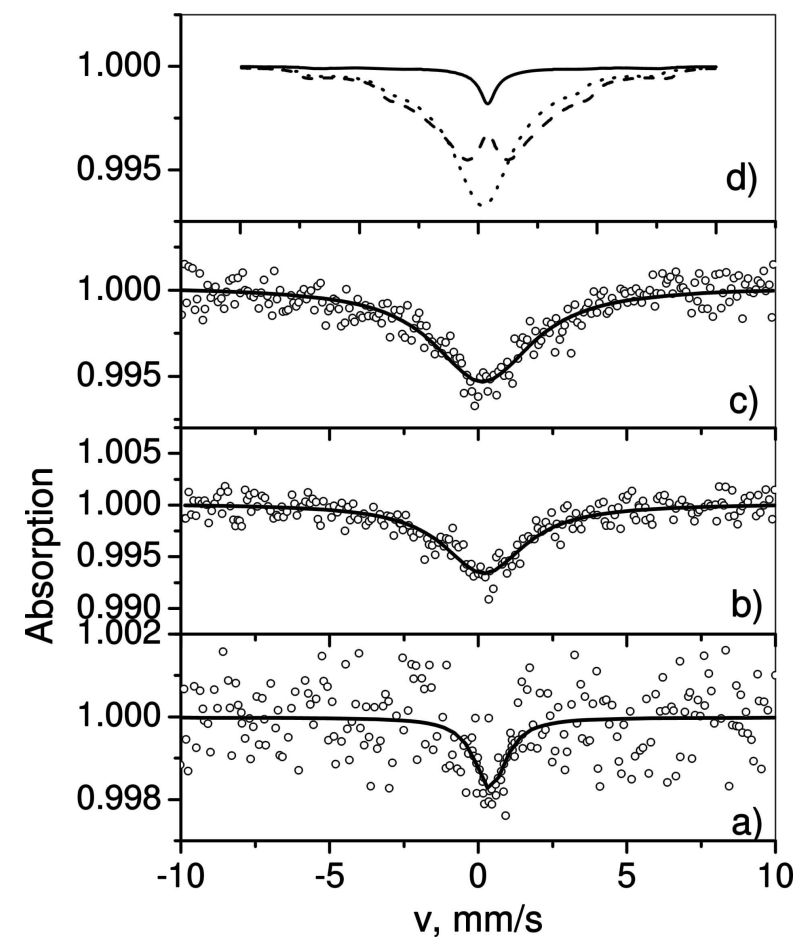

Fig. 2. Mössbauer spectra of $\mathrm{Zn}_{0.5} \mathrm{Co}_{0.5} \mathrm{Fe}_{2} \mathrm{O}_{4}$ particles with the mean diameter of $7.4 \mathrm{~nm}$ at $300 \mathrm{~K}$ : (a) external magnetic field $H$ is $0 \mathrm{~T}$, (b) $H=0.07 \mathrm{~T}$, (c) $H=0.2 \mathrm{~T}$. (d) Simulated spectra using many-state model at $T=300 \mathrm{~K}$ : solid line $H=0$, dashed line $H=0.035 \mathrm{~T}$, and dotted line $H=0.035 \mathrm{~T}$ using the distribution of magnetic fields.

jumping rate between them is rapid, the corresponding lines of spectra are smeared, and the resultant is seen. For example, although $10 \mathrm{~nm}$ size nanoparticles of $\mathrm{Fe}_{3} \mathrm{O}_{4}$ have some thousand of iron atoms, only $S=$ 100 is used in all our simulations. The larger number of states leads to a very similar result.

The Mössbauer spectra of the ferrofluid of $\mathrm{Zn}_{0.5} \mathrm{Co}_{0.5} \mathrm{Fe}_{2} \mathrm{O}_{4}$ particles measured at room temperature are shown in Fig. 2 [11]. The average diameter of particles is $7.4 \mathrm{~nm}$. The ferrofluid is placed into the porous filter paper, which enables preventing the accumulation of particles at poles when the external magnetic field is applied. The application of the filter paper with the pores of some micrometres also allowed increasing the probability of the experimental Mössbauer effect in the sample. The perpendicular external magnetic field of 0.07 and $0.2 \mathrm{~T}$ is applied for the spectra shown in Fig. 2(b,c). The Mössbauer spectra in Fig. 2 can be described by the single Lorentzian line, the width of which increases from 1.3 to $4.0 \mathrm{~mm} / \mathrm{s}$. The increase of the line width is associated with the application of the external magnetic field. A larger than usual line width for the sample, when the magnetic field is not applied, is probably caused by effects of the Brown- ian motion, relaxation, distribution of sizes of particles, and quadrupole splitting.

The relaxation of the magnetic moment can be stopped by the external magnetic field $H$ because of the increase of the barrier. In this case the magnetic energy of the particle is

$$
E=-K V \cos ^{2} \theta-\mathbf{m} \mathbf{H}
$$

where $\mathbf{m}$ is the magnetic moment of the particle. The simplest case of interaction with the external magnetic field would be if the magnetic field were collinear with the easy magnetization axis. Only in this case the many-state relaxation model in form of Eq. (8) can be applied. The equation for the non-collinear magnetic field would be more complicated and Liouville operators would be needed there [10].

The simulated lines in Fig. 2(d) correspond to $H=$ $0 \mathrm{~T}$ (solid line) and $H=0.035 \mathrm{~T}$ (dashed and dotted lines). The value of the magnetic field $H=0.035 \mathrm{~T}$, which can explain additional broadening of the line, is much lower than those applied in the experiment. Therefore, the value of the magnetic field used in simulation can be apparently attributed to the efficient one. When the external magnetic field is applied, the demagnetization field has also to be taken into account. It depends on the sample magnetization and form. In addition, the easy magnetization directions of particles and the magnetic field are not collinear. Therefore, the efficient magnetic field depends on the angle between the easy magnetization axis and the magnetic field. The dotted line in Fig. 2(d) shows the line shape simulated when the efficient magnetic field is distributed according to the Boltzmann law taking into account different directions of the magnetic moment in the external magnetic field. For ferrofluids, the Brownian motion of particles in liquid is important. The Brownian motion rotates the particles. Therefore, the direction of the easy magnetization axis changes with time. The dependence of magnetization of the ferrofluid on external magnetic field is caused by Brownian rotations as well as by thermal fluctuation of direction of the magnetic moment around the easy magnetization axis. From the point of view of Mössbauer spectroscopy, the Brownian rotation, which causes rotation by large angles, is slow. Therefore, it does not directly affect Mössbauer spectra but causes the changes in the barrier height. The rotation by a small angle is faster and therefore can increase the fluctuation rate of the magnetic moment. Such difference in time scales between Mössbauer spectroscopy and the sample magnetization is important in ferrofluid studies. However, a 
strict description of Mössbauer spectra by the manystate model, when non-collinear contributions to the barrier height exist, requires the Liouville operators.

\subsection{Modelling of the average magnetic hyperfine field}

The iron atoms in the lattice may occupy nonequivalent sites which are characterized by the different values of hyperfine parameters of Mössbauer spectra. The example of such material is $\mathrm{Zn}_{x} \mathrm{Co}_{1-x} \mathrm{Fe}_{2} \mathrm{O}_{4}$ nanoparticles, where iron atoms in two different sublattices may have a different amount of $\mathrm{Zn}$ and Co atoms in their neighbourhood. In such cases, Mössbauer spectra are the sums of different subspectra. Frequently, the complicated spectra, which have many different nonequivalent sites, can be expressed by the distribution of hyperfine parameters. For magnetically split Mössbauer spectra, the distributions of the hyperfine field $P(H)$ are obtained, when the spectra are decomposed to a set of subspectra, the hyperfine fields of which differ by an equal amount $\Delta H_{\mathrm{i}}$. Consequently, the average hyperfine field $\left\langle H_{\mathrm{i}}\right\rangle=\sum_{j} H_{\mathrm{i}}^{j} p_{j}$ may be calculated, where $p_{j}$ is the relative area (probability) of the subspectrum $j$. For nanoparticles, the subspectra are affected by relaxation, therefore, the decomposition to Lorentzian subspectra becomes even more complicated because of their overlapping. Despite that, the average value of the hyperfine field $\left\langle H_{\mathrm{i}}\right\rangle$ for the Mössbauer spectrum can always be calculated. Its dependence on temperature will characterize the dependence of the relaxation rate.

For the simulations using the many-state model instead of $\left\langle H_{\mathrm{i}}\right\rangle$, the average splitting of spectra $\langle|v|\rangle$ is more convenient to use. The splitting of the Mössbauer spectrum with the magnetic structure is proportional to its hyperfine field. Therefore, $\left\langle H_{\mathrm{i}}\right\rangle / H_{\mathrm{i} 0}=\langle|v|\rangle / v_{0}$, where $H_{\mathrm{i} 0}$ and $v_{0}$ are the average values of the hyperfine field and the splitting for the static spectrum. The average splitting is evaluated numerically by

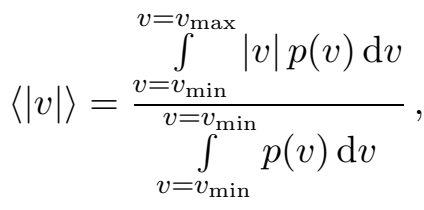

where $p$ is the amplitude of the spectrum at the velocity $v$.

The average hyperfine field $\left\langle H_{\mathrm{i}}\right\rangle$ or $\langle|v|\rangle$ determined from experimental spectra can be compared with the average splitting of Mössbauer spectra obtained by the simulation using the many-state model. Simulated dependences of the average splitting of Mössbauer spectra are shown in Fig. 3. The many-state model $(S=$

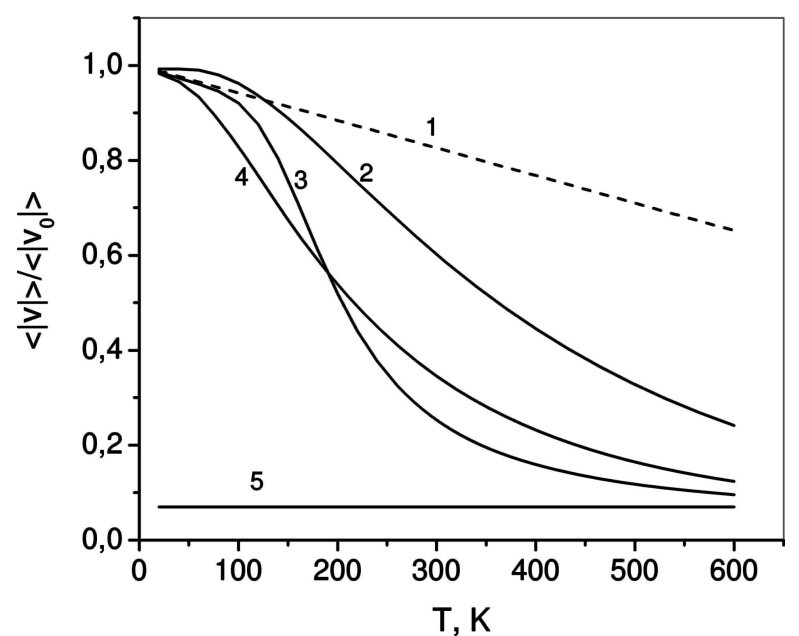

Fig. 3. Simulated relative average splitting of Mössbauer spectra for nanoparticles of diameter $d=7.4 \mathrm{~nm}$ and anisotropy constant $K=5 \cdot 10^{4} \mathrm{~J} / \mathrm{m}^{3}$. 1 marks the dependence according to Eq. (5), 2 is for three-state model $(S=1), 3$ is for many-state model $(S=$ $100)$ with no volume distribution of particles, 4 is for many-state model $(S=100)$ with the standard deviation of the particle size $\sigma=$ $0.6,5$ is for the average splitting of the singlet spectra of width $\Gamma=$ $0.3 \mathrm{~mm} / \mathrm{s}$

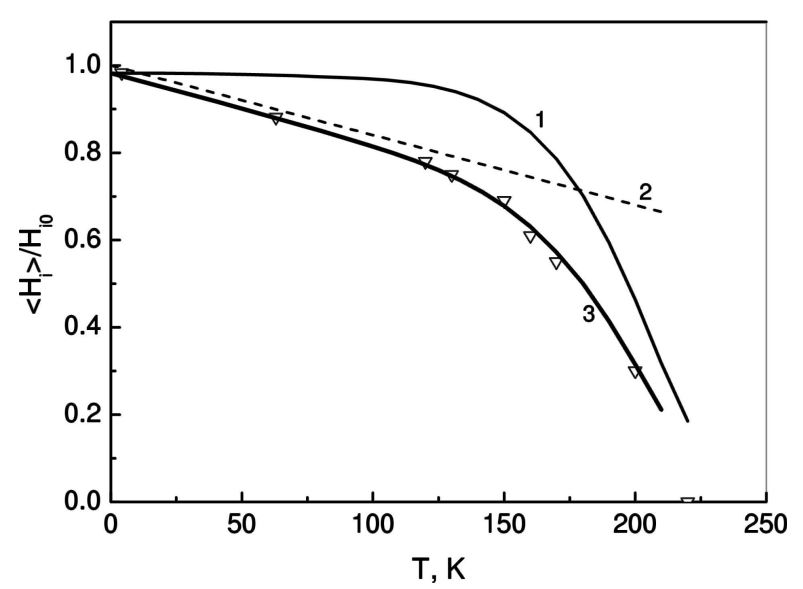

Fig. 4. Dependence of the relative hyperfine field for $\mathrm{Zn}_{0.5} \mathrm{Co}_{0.5} \mathrm{Fe}_{2} \mathrm{O}_{4}$ particles of $7.4 \mathrm{~nm}$ diameter on temperature and their description. 1 is the dependence of relative splitting of Mössbauer spectra, 2 is the dependence of bulk magnetization on temperature, 3 is corrected dependence of relative splitting of Mössbauer spectra.

$100)$ correctly describes the initial linear dependence given by Eq. (5). On the contrary, $S=1$ or a threestate model gives an incorrect result (curve 2 in Fig. 3). It is because the three-state model does not take into account the deviations of the magnetic moment of the particle by small angles. The simulation of the average splitting of spectra $\langle|v|\rangle$ has shown the importance of the influence of the size distribution of nanoparticles. The dependence of $\langle|v|\rangle$ on temperature deviates from the initial linear law (Eq. (5)) at lower temperatures, 


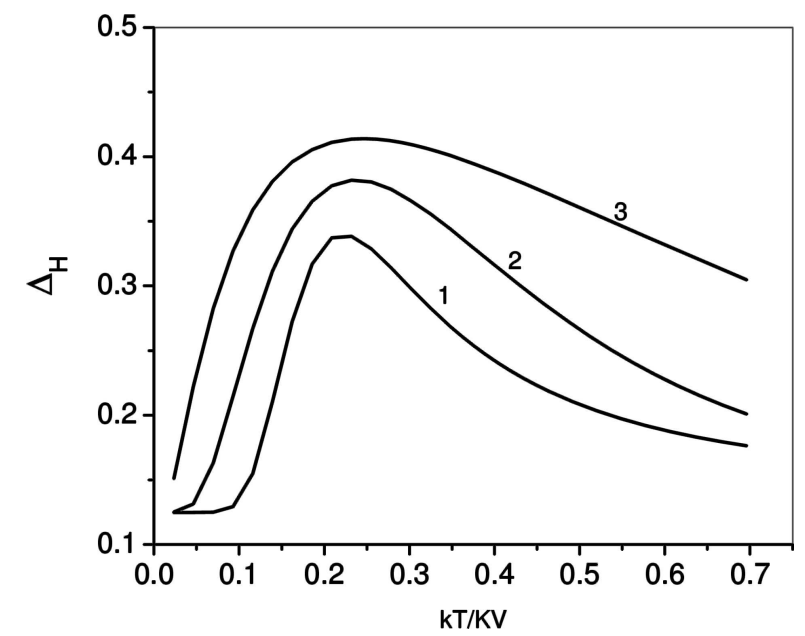

Fig. 5. Dependence of deviation $\Delta_{H}$ of the hyperfine field from its average on normalized temperature when $(1)$ deviation of the particle diameter is $\sigma=0$, (2) $\sigma=0.5,(3) \sigma=1$.

when the volume distribution of particles is broader, due to the particles smaller than average. It is usually assumed that Eq. (5) is valid when the hyperfine field decreases not more than by $8-10 \%$.

The simulation of average splitting of spectra using the many-state model is applied for the description of experimental data [11] for $\mathrm{Zn}_{0.5} \mathrm{Co}_{0.5} \mathrm{Fe}_{2} \mathrm{O}_{4}$ particles of the average $7.4 \mathrm{~nm}$ diameter (Fig. 4). It is seen that at low temperature the dependence of the hyperfine field on temperature almost coincides with the dependence of bulk magnetization. This means that the anisotropy barrier $(\Delta E=K V)$ is sufficiently high and the influence of relaxation of the magnetic moment around the easy magnetization axis is negligible. Thus, the linear dependence of the hyperfine field is due to the decrease in bulk magnetization and not due to the increase of the angle of deviations of the magnetic moment direction. At higher temperatures, $\left\langle H_{\mathrm{i}}\right\rangle$ starts to decrease more rapidly, which is already caused by the increase of the rate of relaxation. The correct description can be obtained when the anisotropy constant decreases with temperature as given by

$$
\begin{aligned}
K(T)= & 7.4 \cdot 10^{4} \frac{\mathrm{J}}{\mathrm{m}^{3}} \\
& +1.5 \cdot 10^{3} \frac{\mathrm{J}}{\mathrm{m}^{3} \mathrm{~K}}[180 \mathrm{~K}-T] .
\end{aligned}
$$

Usually from the Mössbauer data for non-interacting particles the blocking temperature is determined. From the blocking temperature the barrier height at blocking temperature can be obtained. The blocking temperature means the temperature when the fast relaxation starts and it is approximately equal to the temper- ature when the magnetic split and paramagnetic parts of Mössbauer spectra are equal. The application of the many-state model in this case shows that it can be used to obtain much more information than there can be obtained from the blocking temperature.

In addition to the average hyperfine field $\left\langle H_{\mathrm{i}}\right\rangle$, the distribution of the hyperfine field can be characterized by deviation of the hyperfine field from its average

$$
\begin{aligned}
\Delta_{H} & =\frac{\sqrt{\left\langle\left(H_{\mathrm{i}}-\left\langle H_{\mathrm{i}}\right\rangle\right)^{2}\right\rangle}}{H_{\mathrm{i} 0}} \\
& =\frac{\sqrt{\left\langle(|v|-\langle|v|\rangle)^{2}\right\rangle}}{v_{0}} .
\end{aligned}
$$

The dependences of $\Delta_{H}$, which is induced by the distribution of sizes, are shown in Fig. 5. The experimental deviation $\Delta_{H}$ can be compared with the simulated one. This can provide additional information on the distribution of the parameters affecting Mössbauer spectra.

\section{Conclusions}

It has already been shown that the many-state model can be used to describe Mössbauer spectra within a wide temperature range even if the external magnetic field is applied. In this study the dependence of the average hyperfine field on temperature is described using the many-state relaxation model. Such simulations allow obtaining more information than it can be derived from the evaluation of the blocking temperature for ferrofluids. The energy of the magnetic moment of particles depends on the angle, magnetic anisotropy, and the magnetic field. In the case of the slow relaxation rate (small deviation angles of the magnetic moment), the form of such dependence at energy minima is important. On the contrary, the fast relaxation is affected mainly by the energy barrier height between the energy minima. The many-state model allows taking into account all such features. In the study the simplest case of uniaxial anisotropy, which is suitable for noninteracting nanoparticles in ferrofluids, is considered. Here, the dependences of splitting of simulated spectra are used to explain the experimental dependence of the average hyperfine field on temperature. As result of fitting of the simulation curve to the experimental data, the dependence of the magnetic anisotropy constant on temperature is obtained.

One of main problems of ferrofluids to be solved is the evaluation of the influence of interaction between the particles, when they aggregate or not, and when 
the external magnetic field is applied or not. In these cases, the many-state model also offers much advantage. Though in cases of interacting particles or the presence of the additional magnetic field, Liouville operators in the many-state model should be introduced.

\section{References}

[1] P. Moriarty, Nanostructured materials, Rep. Prog. Phys. 64, 297-381 (2001).

[2] B. Huke and M. Lücke, Magnetic properties of colloidal suspensions of interacting magnetic particles, Rep. Prog. Phys. 67, 1731-1768 (2004).

[3] M.F. Thomas and C.E. Johnson, in: Mössbauer Spectroscopy, eds. D.P.E. Dickson and F.J. Berry (Cambridge University Press, Cambridge, 1986) pp. 143197.

[4] S. Dattagupta, in: Mössbauer Spectroscopy, eds. D.P.E. Dickson and F.J. Berry (Cambridge University Press, Cambridge, 1986) pp. 198-218.

[5] W.F. Brown, Thermal fluctuation of a single-domain particle, Phys. Rev. 130, 1677-1686 (1963).
[6] A. Aharoni, Thermal agitation of single domain particles, Phys. Rev. 135, A447-A449 (1964).

[7] D.H. Jones and K.K.P. Srivastava, Many-states relaxation model for the Mössbauer spectra of superparamagnets, Phys. Rev. B 34, 7542-7548 (1986).

[8] J. van Lierop and D.H. Ryan, Mössbauer spectra of single-domain fine particle systems described using a multiple-level relaxation model for superparamagnets, Phys. Rev. B 63, 064406-1-8 (2001).

[9] H.D. Pfannes, J.H. Dias Filho, R. Paniago, and R. Magalhaes-Paniago, Simulation of Mössbauer spectra of superparamagnets under external fields using a many-state model, Hyperfine Interactions 141/142, 193-198 (2002).

[10] M. Blume, Stochastic theory of line shape: Generalization of the Kubo-Anderson model, Phys. Rev. 174, 351-358 (1968).

[11] A. Amulevičius, D. Baltrūnas, A. Daugvila, P.C. Fannin, Yu.D. Perfiliev, D.A. Pankratov, and A. SlawskaWaniewska, Mössbauer study of mixed ferrite ferrofluids, Bull. Russ. Acad. Sci. 67, 1160-1164 (2003).

\title{
HIPERSMULKIOJO MAGNETINIO LAUKO NANODALELĖSE MODELIAVIMAS
}

\author{
K. Mažeika, A. Amulevičius, D. Baltrūnas
}

Fizikos institutas, Vilnius, Lietuva

\section{Santrauka}

Tiriant magnetinių momentų dinamiką nanostruktūrinėse magnetinèse medžiagose, Mesbauerio (Mößbauer) spektrai turi būti aprašomi tinkamais dinaminiais relaksaciniais modeliais. Nagrinèjamos tam tikslui skirto daugelio būsenų relaksacinio modelio galimybès. Toks modelis gali visapusiškai paaiškinti feroskysčiu spektrų priklausomybę nuo temperatūros. Sudètingesnių medžiagu
Mesbauerio spektrams, kuriuose yra daugiau negu vienas statinis pospektris, tinkamesnis kitimo nuo temperatūros apibūdinimas yra panaudojant magnetinio hipersmulkiojo lauko vidurkị. To lauko vidurkio priklausomybės nuo temperatūros modeliavimas daugelio būsenų modeliu leidžia ìvertinti parametru (pvz., magnetinès anizotropijos), itakojančiu magnetinio momento relaksaciją, priklausomybę nuo temperatūros. 